\title{
DimSUM: Dimension and Scale Unifying Map for Visual Abstraction of DNA Origami Structures
}

\author{
H. Miao, ${ }^{1,2}$ E. De Llano, ${ }^{2}$ T. Isenberg, ${ }^{3}$ M. E. Gröller, ${ }^{1,4}$ I. Barišić, ${ }^{2}$ and I. Viola ${ }^{1}$ \\ ${ }^{1} \mathrm{TU}$ Wien, Austria \\ ${ }^{2}$ Austrian Institute of Technology, Austria \\ ${ }^{3}$ Inria and Université Paris-Saclay, France \\ ${ }^{4}$ VRVis Research Center, Austria
}

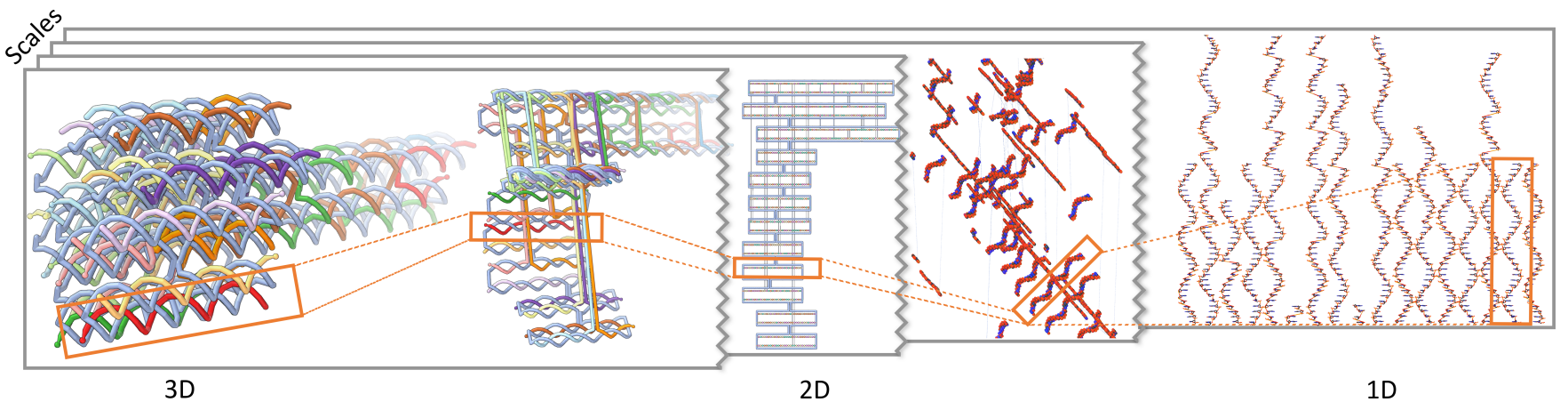

Figure 1: A nanotube transitions from a $3 D$ shape model to a diagrammatic $2 D$ representation and finally to a clean $1 D$ alignment of single strands. Orthogonal to the dimension axis is the scale axis, which shows different semantic scales. The orange rectangle tracks a single strand through the dimensions and scales.

\begin{abstract}
We present a novel visualization concept for DNA origami structures that integrates a multitude of representations into a Dimension and Scale Unifying Map (DimSUM). This novel abstraction map provides means to analyze, smoothly transition between, and interact with many visual representations of the DNA origami structures in an effective way that was not possible before. DNA origami structures are nanoscale objects, which are challenging to model in silico. In our holistic approach we seamlessly combine three-dimensional realistic shape models, two-dimensional diagrammatic representations, and ordered alignments in one-dimensional arrangements, with semantic transitions across many scales. To navigate through this large, two-dimensional abstraction map we highlight locations that users frequently visit for certain tasks and datasets. Particularly interesting viewpoints can be explicitly saved to optimize the workflow. We have developed DimSUM together with domain scientists specialized in DNA nanotechnology. In the paper we discuss our design decisions for both the visualization and the interaction techniques. We demonstrate two practical use cases in which our approach increases the specialists' understanding and improves their effectiveness in the analysis. Finally, we discuss the implications of our concept for the use of controlled abstraction in visualization in general.
\end{abstract}

CCS Concepts

•Human-centered computing $\rightarrow$ Scientific visualization; Visualization theory, concepts and paradigms; •Applied computing $\rightarrow$ Computational biology;

\section{Introduction}

DNA nanotechnology is a young yet rapidly progressing field that aims to design nanoscale devices by employing DNA as the main building block [See82]. The great chemical stability of DNA and the availability of synthetic DNA production facilities make it a preferred material for intricate nanostructures. The resulting, increasingly complex nanoscale shapes show great potential in medicine and biotechnology. DNA nanotechnology exploits the base pairing capability of DNA and the synthesis of short staple strands to fold the long scaffold strand (hence the name origami). DNA origami is a widely established method for creating DNA-based nanoscale shapes as introduced by Rothemund [Rot06].

For this application domain we designed and developed an interactive data exploration and modeling approach that relies on a 
two-dimensional abstraction space. It spans multiple conceptual scales as well as multiple spatial dimensions for the data representation (Fig. 1). We use it as a navigational map to select the best suitable visual representation for a specific task (Fig. 2). With this concept we go well beyond the approach taken in the domain's popular software tool caDNAno [CD16], which implements functionality of the DNA origami method and enables scientists to design nanoscale shapes in silico. However, advanced computational DNA design concepts are needed, as the current tools are not designed for the ever-increasing complexity of DNA nanostructures. Available tools currently only support the design of 3D shapes on 2D DNA diagrams. As a result, the diagrams exhibit a high degree of clutter and overlapping edges. Although domain scientists employ different representations of these complex structures, they are not able to mentally link between them visually. The resulting cognitive load on their workflow increases the overall duration of designing intricate nanostructures and decreases the confidence in their in silico design. Moreover, undiscovered errors in the design can lead to failed experiments, which can take from several weeks to months until the self-assembled structures are inspected in a microscope. As a consequence, these problems in the in silico design currently slow down the research progress.

With our 2D abstraction map DimSUM, we integrate and visually link the representations of the 3D target structure, the 2D diagrams, as well as $1 \mathrm{D}$ arrangements to enable designers to effectively apply manipulations in the dimension that is most suited. With DimSUM we also integrate seamless transitions that allow researchers to mentally link different representations. With this multi-scale and multi-dimensional approach we thus facilitate researchers to gain more confidence in the design and in the end enable them to create increasingly more intricate structures. They can effectively analyze and understand the structural properties of these complex objects, to gain confidence in the in silico design before carrying out the experiment in the laboratory itself. Ultimately this significantly reduces the time and costs needed for typical design operations.

Our interactive visualization design is based on a close collaboration with an interdisciplinary team that aims to create a DNA nanorobot, which consists of several complex DNA origami components. We started by analyzing the requirements and the needed visual representations of this domain. Based on this analysis, we extended a previous multi-scale concept [MDLS*18], which only abstracts the 3D structure on one scale-axis. Indirect 2D and 1D representations are widely used in the domain, due to their occlusion free layout, but they are not suited for estimating the actual 3D shape of the structure. Therefore, we integrate the 2D and 1D representations into a comprehensive and novel abstraction space, spanned by a scale and a dimension axis. Using our resulting abstraction map DimSUM as a tool to navigate the different representations, we designed a number of additional interaction techniques that support domain scientists in their tasks. We illustrate these practical tasks needed by our collaborators, using several design studies. Our contributions are as follows:

- An integration of novel and existing DNA nanostructure representations into an interactive abstraction map that allows domain scientists to perceive and understand the conceptual space and geometric relationships between established and new DNA nanos-

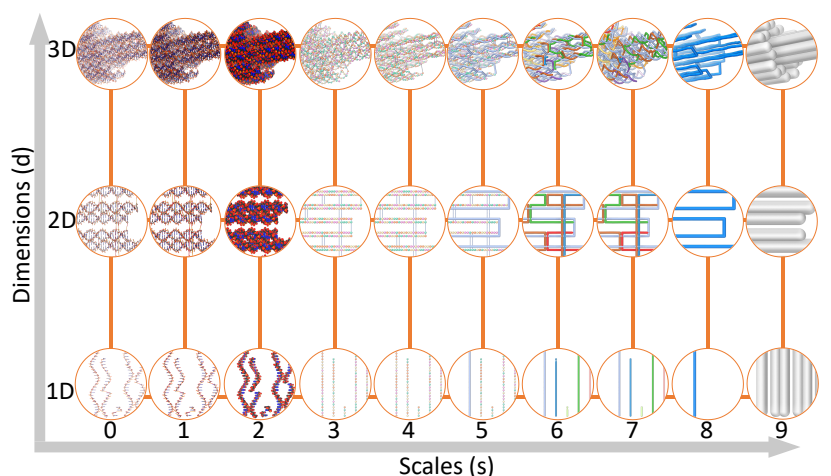

Figure 2: The DimSUM abstraction map is spanned by a dimension and a scale axis and integrates existing and novel representations.

tructure representations. We extend the multi-scale approach of Miao et al. [MDLS*18] with an orthogonal dimension axis to utilize the strength of 2D and 1D layouts to analyze, make sense of, and especially modify the complex structures.

- With this design, we advance the discussion of the concept of abstraction in illustrative visualization. We demonstrate that the spatial layouting of the visual elements along the dimensionality is an important axis of abstraction. The axis goes from a realistic shape depiction to a successfully simpler representation of the elements where alignment details are gradually abstracted.

- We integrate a number of specific interaction techniques that support the DimSUM design including abstraction axis snapping, guiding (heat-)maps, and saving viewpoints to support both navigation of the abstraction map as well as the analysis of taskspecific interaction behaviors.

\section{Background: DNA Origami}

DNA origami [Rot06] relies on the self-organizing nature of DNA molecules to construct nanoscale objects. The method uses one long (approx. 8000 bases) Single Stranded DNA (ssDNA) and numerous shorter (20-80 bases) synthetic ssDNA strands. These shorter staple strands bind to specific regions on the long scaffold strand to fold it together into a targeted shape. A strand has a directionality and it goes from the 5' to the 3' end of the nucleotide sugar ring, which is relevant for the anti-parallel pairing of ssDNA. DNA nanotechnology exploits the base pairing capability of DNA, i. e. two strands only pair and form Double Stranded DNA (dsDNA) if the sequence of nucleotides are complementary to each other. The Watson-Crick complements [WC53] describe that Adenine (A) only pairs with Thymine (T) and Cytosine (C) pairs only with Guanine (G). The helices are then held together by crossovers at designated positions, where one ssDNA switches from one helix to an adjacent one. Constraining the adjacent helices to potential crossover locations results in a regular pattern of dsDNA alignment, which facilitates the creation of stable structures. Based on this, Douglas et al. [DMT*09] proposed two lattices, a honeycomb and a square lattice, for their caDNAno tool.

The DNA used in these experiments is typically described by different structural representations as shown in Fig. 3. Scientists thus have to consider each representation carefully when designing 


\section{H. Miao \& E. De Llano \& T. Isenberg \& M. E. Gröller \& I. Barišić \& I. Viola / DimSUM: Dimension and Scale Unifying Map}

these nanostructures. The primary structure is determined by the sequence of ssDNA. It is usually depicted as a string of bases from the 5' to the 3' direction, which motivates the $1 \mathrm{D}$ view we use in our representation later-on. The DNA's self-assembly is determined by the sequence of the scaffold and staple strands. The scientists need abstract representations, which allow them to inspect aspects of the individual strands. For example, long staple strands ( $>80$ bases) are increasingly costly to synthesize. In addition, the occurrence of $\mathrm{C}$ and $\mathrm{G}$ on a strand have to be carefully considered as they tend to be stickier than other nucleotides. Finally, a high frequency of crossovers per strand could have an adverse effect on the stability of the structure. All of these analysis steps require a separate view on each staple strand, yet in the state-of-the-art-tools like caDNAno the strands are interwoven in 2D DNA diagrams.

We employ 2D diagrams to show the secondary structure, which describes the base paring interaction of the ssDNA, that forms the dsDNA. The 2D view indicates adjacencies of the double helices by placing the double strands underneath each other. This $2 \mathrm{D}$ layout for the design of a 3D structure results in many connections between adjacent double strands in 3D that are not neighbors in the 2D diagram, cluttering it with many overlapping edges as the structure grows in complexity (see the example in Fig. 3). The resulting diagram is difficult to understand-in a situation where a correct design is crucial for the success of experiments.

The three-dimensional shape of the target structure, finally, results from the tertiary structure that encodes the spatial 3D model. This motivates our use of an additional 3D representation. The correct folding of the scaffold strand, for example, can only be achieved if the spatial properties of the strands are carefully considered. The exact folding can only be seen by imaging the results of in vitro experiments - the best in silico predictions of foldings are computationally very expensive and have limitations. An approximate $3 \mathrm{D}$ representation of what the nanostructure would look like if it were to fold properly, however, already allows the domain experts to study the properties on the model. Another advantage is that distances can be estimated in a 3D spatial model - a crucial task for the domain scientists. The combination of this model with already tested design techniques increases the confidence in the in silico constructions.

\section{Related Work}

Our work builds on concepts and methods in DNA nanostructure visualization, visual abstraction, and $\mathrm{HCI}$, which we review below.

DNA nanostructure visualization: For our visualization concept, we integrate existing as well as several novel representations. Among the former, diagrams have been used by domain researchers as simplified DNA representations for a long time. In Rothemund's work on DNA origami [Rot06], for instance, dsDNA is depicted in an unrolled way, showing it as colored arrows to indicate the ssDNA and their crossovers. Douglas et al. [DMT*09] proposed a tool to rapidly prototype DNA origami structures based on a similar representation. In addition, he depicts the arrangement of the dsDNA on a lattice that shows the structure from an orthogonal direction Based on the regular appearance of potential crossover positions on the dsDNA, this representation leads to a hexagonal honeycomb lattice and a square lattice. In addition, a view of the target 3D form helps the user to understand the final shape of the structure. Using an alternative representation, Benson et al. $\left[\mathrm{BMG}^{*} 15\right]$ described a technique to semi-automatically model polyhedral nanostructures in 3D space, representing nucleotides as spheres. For both types of representations the authors have made well-motivated design decisions that allow the users to build the desired target shape. Various structural details are not described, which are necessary for advanced tasks such as structural modifications of functional nanostructures The 2D diagrams, for example, are not well suited for tasks in which spatial features have to be considered. Nonetheless, the 2D diagrams by Douglas et al. [DMT*09] are widely known in the field of DNA nanotechnology. We thus integrate them as part of our $2 \mathrm{D}$ view, and extend them with seamless transitions to other representations of different dimensionality such as $3 \mathrm{D}$ views.

Visual abstraction: The visual abstraction of spatial data is a core concept of illustrative visualization [VI18] that has been applied in numerous domains. ${ }^{\dagger}$ For example, the schematization of network data such as streets has been studied in detail, both for traditional geography applications [CdBvK05] and for artistic purposes [Ise13]. A continuous transition of different presentation styles of city models based on task, camera view, and image resolution has been proposed by Semmo et al. [STKD12]. Researchers have also investigated the non-uniformly controlled adjustment of abstraction [AS01] to support, in particular, navigation. Similar to the linear structures in cartography, the DNA strands in our application domain exhibit information that is not always relevant. As an example, for certain analysis tasks the twist of the double helix or the pathway might be irrelevant. In our 1D view we thus straighten the DNA strands and indicate crossover locations-in a way similar to subway lines and maps which share similar characteristics to preserve topological information [Rob12]. For this representation we are also inspired by the straight alignment of single tracks as proposed by Wu et al. [WTLY12] for the purpose of customizing travel paths.

A core advantage of visual abstraction is that it can preserve a visual variable or the (screen) space for the depiction of additional data [VI18]. Cipriano and Gleicher [CG07], for example, abstracted the molecular surface while keeping significant shape features, allowing them to place glyphs on the surface to encode additional properties. We use the same principle in our work to depict data, for instance, about the stability of the DNA origami structures.

A lot more recent work on visual abstraction concerns the dedicated control of the amount of abstraction to support various visualization purposes. One way to control the degree of abstraction is to show different representations of the same object, depending on the distance to the viewer. Parulek et al. [PJR $\left.{ }^{*} 14\right]$ demonstrated this approach also for molecular data, where it serves the visual comprehension and also makes it possible to visualize complex biological environments at interactive rates [LMAPV15]. In addition to such an application, controlled abstraction can also support the exploration of different semantic scales. Miao et al. [MDLS*18], integrated several representations of DNA nanostructures and arranged them

\footnotetext{
$\uparrow$ For a more complete survey of related work on visual abstraction we refer to Viola and Isenberg's recent survey/meta paper on the subject [VI18]. Here we only point out aspects that closely relate to or inspire our own work.
} 
on an axis of semantic abstractions that smoothly controls the representation. We use their linear interpolation-based transition and adapt the ten semantic scales to our 2D and 1D layouts. We add the abstraction along different spatial dimensions to facilitate a much more comprehensive exploration of DNA origami structures that also integrates 2D schematics and 1D alignments of DNA strands.

This process leads to the creation of a multi-dimensional abstraction space [VI18] that can be used for the exploration of visual structures by assisting users to mentally integrate different representations. Zwan et al. [vdZLBI11] were the first to construct a 3D abstraction space with several components: structural abstraction ('geometric abstraction' in Viola and Isenberg's terminology), abstraction of the illustrativeness, and different degrees of support of spatial perception (the latter two are 'photometric abstractions' in Viola and Isenberg's terms). Recently, Mohammed et al. [MAAB*18] discussed a similar concept for the controlled abstraction of connectomics data. They constructed a 2D abstraction space, the first axis depicting representations of astrocytes and the second axis depicting neurites. In contrast to their work, our approach is more general and not limited to two structures, as our abstraction map is spanned by different axes of abstraction (scale and dimension).

While we employ a similar interaction concept in our work, we augment the novel DimSUM abstraction map with specific interaction techniques that specifically support navigation. Furthermore, we enable viewers to control the transition between 1D, 2D, and $3 \mathrm{D}$ space without constraints. The dimensional transitions act as dedicated illustration tools to support the understanding of the relationships between the dimensions.

Interaction: The interaction facilitated by the DimSUM widget relates to the concept of multiple coordinated viewss (MCVs) [BC87, BMMS91, Rob07, Wil08]: Users interact with the presented data and visually inspect them through different views that are linked to each other. However, our domain users require operations on many scales, dimensions, and their intermediate transition states. Having many views using MCVs would not be feasible, as the single views would get too small. The DimSUM widget provides a different interaction concept as it allows users to transition between representations shown in another view. Our interaction is thus more akin to approaches like Jianu et al.'s [JDL09] exploration of three-dimensional brain fiber tracts using a previously generated abstract $2 \mathrm{D}$ representation. We use animations to transition between the differently abstracted representations. Animation is shown to facilitate comprehension, learning, and memory communication by Tversky et al. [TMB02] and has been previously applied for molecular abstractions by Sorger et al. [SMR*17]. In addition, we also employ brushing and focusing [BMMS91] for selecting elements and observing their transition through dimensions and scales.

\section{Methodology and DimSUM Concept}

Our work has been motivated by collaborations with a team of DNA nanotechnology researchers whose ambitious goal is to create a celldrilling nanorobot. The first author $(\mathrm{C} 1)$ of this paper, a visualization researcher, has been working for 11 months as a member of the nanotechnology team and works $50 \%$ of his time at their lab. The team is led by the Principal Investigator (C2) who develops innovative concepts of parts of the nanorobot. The existing tools in the DNA nanotechnology domain to support such complex work are reaching their limits. This is the case in terms of functionality and especially in terms of available visual representations for the features of these structures. From early-on, many challenges emerged regarding the visualization and modeling of the involved complex structures.

C1 was thus asked to develop a new visualization concept and, ultimately, a system required for reaching the goal of creating functional DNA nanorobots using stable DNA origami structures. Based on observations, interviews, and focus group discussions, $\mathrm{C} 1 \mathrm{ex}-$ tracted the necessary context and challenges (Sect. 2). Together with the entire team, he derived the tasks and the design considerations for the visualization system. For example, domain scientists analyze different structural properties using different layouts, as described in Sect. 2. Then, they would like to directly examine and manipulate the structure in the appropriate layout. $\mathrm{C} 1$ also extensively collaborated with a physicist (C3), and $\mathrm{C} 1$ and $\mathrm{C} 3$ were responsible for developing a novel software system required for reaching the goals of the ambitious nanorobot project. In addition, a biochemist (C4) focused on the design of DNA origami structures, while another molecular biologist (C5) was specializing in the creation of novel functional structures. C4 and C5 were also responsible for carrying out the experiments and assembling the designed nanostructures in the laboratory, after the in silico design was finished.

Depending on the scientists' subgoals and tasks, they use different representations for modeling, editing, analyzing, and creating conceptual designs. For instance, $\mathrm{C} 2$ and $\mathrm{C} 4$ heavily relied on 2D diagrams where the structure can be quickly modeled. This work requires a deep understanding of the relationship between the $2 \mathrm{D}$ diagrams and the associated 3D model-yet they had no direct way of manipulating the structure in 3D space. $\mathrm{C} 5$ depended on having a detailed view on the atomic configuration for changing nucleotide conformations at an atomic level with a 3D spatial model. Since he collaborated with $\mathrm{C} 4$ for trying to connect the different components together, he needed a way to understand the newly designed structure in 2D as well. This integrated handling of representations across spatial dimensions is not possible with existing tools. The generation of functional structural motifs required C3 to understand the relationship between the conformation of atoms and the twists of the double helices. For scaffold routing, she did not require a realistic spatial model, but needed to see the entire structure in 2D and without visual occlusion. To evaluate the potential crossovers, she relied on analyzing potential crossover locations in a 3D view and also on being able to manipulate the structure in the same view. After finalizing the design, the experts exported the sequence of single strands as a simple sequence of the bases (Fig. 3). While it was common that this sequence of strands would still be modified, there was no way to relate these changes back to the initial 2D or 3D representations. In summary, the current in silico design process is inflexible and complex. New visualization technology that integrates the different representations is urgently needed.

\subsection{DimSUM: Integration of Dimension and Scale}

As caDNAno is the state-of-the-art tool to create DNA origami structures, we focused on its data representations. We demonstrate our design concept on a prototypical nanotube, consisting of a smaller 


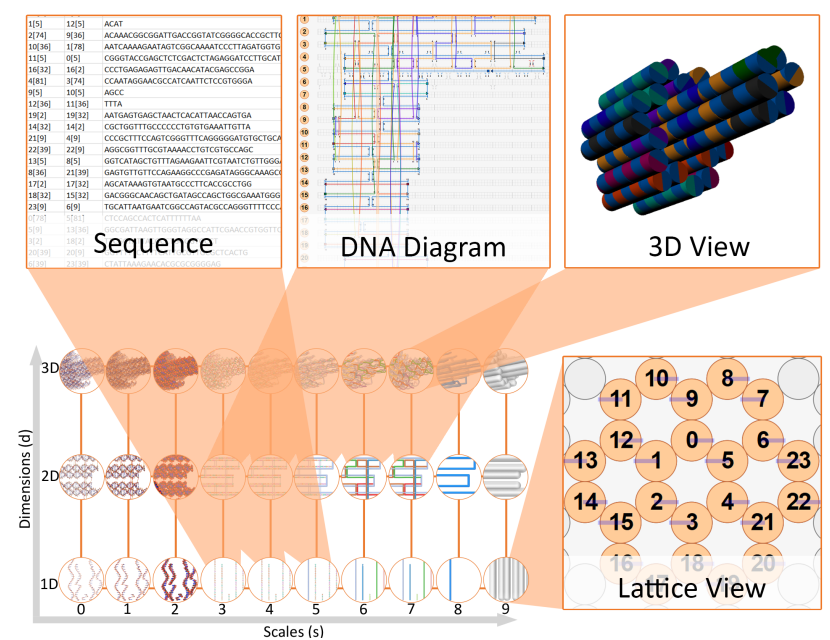

Figure 3: We provide many ways of interacting with and seamlessly transition between multiple structural representations using the DimSUM. Here we show which parts of the abstraction map depict equivalent content as the state-of-the-art tool caDNAno.

shaft and a wider body, as shown in Fig. 4. We used caDNAno's automated staple placement to create it. The scientists did not apply any further modifications, so that we can demonstrate the use of our approach to analyze and improve the structure. The nanotube has a length of $260 \AA$ and a diameter of $50 \AA$ at the shaft and $120 \AA$ at the body. The scaffold is 1003 nucleotides long and the structure is folded by 24 staple strands. In Fig. 3 we compare the views that caDNAno provides and show how we integrated equivalent ones into our DimSUM abstraction space. Each of the four boxes depicts a view provided in caDNAno. We isolate the different spatial dimension $(d)$ of the representations and treat them as one axis of our abstraction space. In addition, we also use different semantic scales $(s)$ of DNA nanoscale structures [MDLS*18] as a second abstraction axis. By arranging the existing representations appropriately and adding the missing ones, the two axes create a unifying, continuous space to access and interpolate between representations (Fig. 2). We first describe the three different spatial dimensions. Afterwards we discuss the transitions between them and interaction mechanisms to assist in the navigation.

3D Representations. We based our three-dimensional representations on Miao et al.'s [MDLS*18] continuous sequence of multiple semantic representations of DNA nanostructures. These representations range from concrete (all-atoms) to abstract (double strands). We extended their concept by more accurately representing the atoms as spheres with van der Waals radii, encoding the $5^{\prime}$ to $3^{\prime}$ direction of ssDNA in Scale 5 and 6, and representing the strand in the most abstract scale as a tube with a realistic radius of $10 \AA$, which the dsDNA occupies (Fig. 4).

These 3D representations realistically encode the spatial arrangement of the DNA origami model after self-assembly. It is important to assess the final overall design (i. e., size and space that the structure occupies) but also to estimate the distances between nucleotides. The multiple scales allow the scientists to visualize the structure without distracting clutter of the 2D views. Most importantly, they

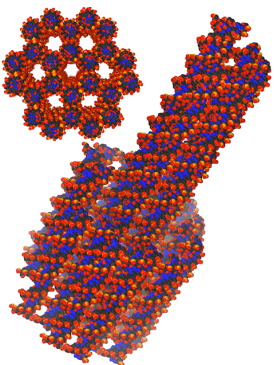

(a) $d=3.0, s=2.0$

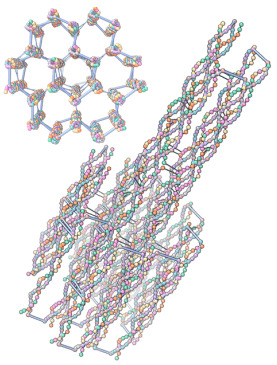

(b) $d=3.0, s=4.5$

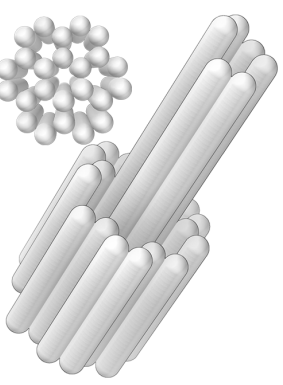

(c) $d=3.0, s=9.0$
Figure 4: Cylindrical nanotube: examples of representations in $3 D$, i.e., $d=3.0$ and variable scales $s$.

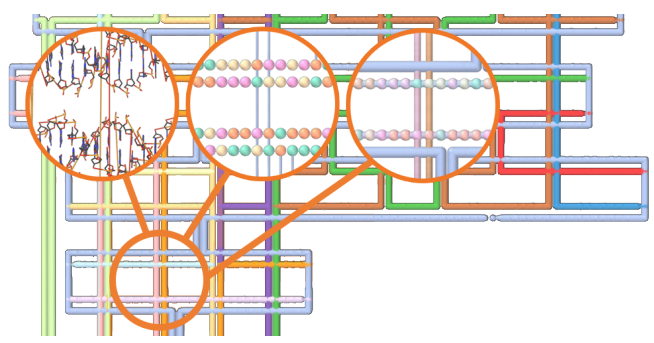

Figure 5: The 2D diagrammatic representation and several examples of views are shown where $d=2$.

are not required to mentally link to the 3D spatial model from a $2 \mathrm{D}$ view when applying modifications because they are able to edit directly in 3D space.

2D Representations. Abstract DNA diagrams are widely employed in the domain due to their visual occlusion-free layout. The arrangement in 2D facilitates an overview of the entire structure. In contrast to the existing representations, we use the same semantic scale representations as in 3D space (Fig. 2) so that the scientists can choose the scale most appropriate to their tasks (e. g., Fig. 5).

Equivalent to the 3D representation, the grey tube depicts the scaffold strand and the colored tubes represent staple strands. In an alternating manner, the staple strands are placed underneath or above the scaffold strand with a distance of $5 \AA$. The vertical distance between the double strands is now $20 \AA$ which guarantees that the complementary strands are spatially close to each other.

With this approach we distribute the complexity of traditional diagrams among several semantic scales. Depending on the scale, we allow the scientists to view detailed features such as atoms and bond conformations in $2 \mathrm{D}$ or to simplify the ssDNA to colored tubes. In addition, the depiction of complementary strands in parallel, without the helicity of the strands, is automatically provided by our semantic abstraction in Scales 3-7. This feature allows the user to get a quick glance of the sequence of a strand and its complementary counterpart. Except for translations in one plane and zooming into details, the user can thus explore the entire structure without having to change the view angle to account for visual occlusions.

1D Representations. After the 2D representations have already removed parts of the spatial context in favor of schematization, 1D 


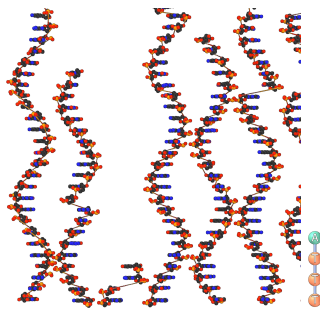

(a) $d=1.0, s=1.5$

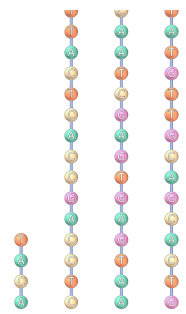

(b) $d=1.0, s=3.0$

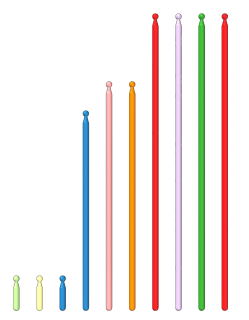

(c) $d=1.0, s=7.0$

Figure 6: Examples of representations in $1 D$, shown at different scales and sorting by (a) strand ID, (b) GC content, and (c) length.

representations only provide a list of straightened single strands. The strands are vertically arranged, aligned side-by-side with a distance of $20 \AA$. This abstract depiction is motivated by the last step of the in-silico design workflow, in which the sequence of the strands are simply depicted as linear strings as shown in Fig. 3, which allows scientists to analyze their individual properties as well as the overall list of strands needed for synthesis and extraction in order to build the structure in vitro.

With the strands aligned straight, it also becomes possible to sort them by certain properties, as shown in Fig. 6. A straightforward sorting is by length. The length of strands is of interest to our domain scientists as it allows them to identify strands with unusual lengths, which cause high costs in the synthesis as described in Sect. 2. The advantage of the DimSUM abstraction map is that it allows the viewer to relate the sorted strands back to the $2 \mathrm{D}$ and $3 \mathrm{D}$ representations. Furthermore, we enable the sorting of strands by their respective content of $C$ and $G$ with respect to the length which indicates the stickiness of a single strand. Such stickiness can either be a desired or an undesired property, depending on the purpose of the staple strand and its placement in the design. The melting temperature and Gibbs free energy can be computed for the binding regions of the strands as done by Miao et al. [MDLS*18], using the thermodynamical model proposed by SantaLucia et al. [SH04]. These two properties can also be used for sorting, roughly indicating the stability of the strands based on their secondary structure. Sorting the strands by the accumulated stability thus enables the user to quickly spot the strands that could compromise the self-assembly, as they pose the weakest link in the chain.

\subsection{Seamless Transitions Across Dimensions and Scales}

To effectively work with the representations, the scientists have to mentally link the representations. To externalize this mental linking, we support control the abstraction and representation process through seamless transitions. We co-register the data and linearly interpolate the positions of elements to guarantee a uniform change between two adjacent representations. In addition, we combine the position-based transformation with the interpolation of shape and color across the scales. There is no rotational or scaling component in the interpolated transformation.

Fig. 1 demonstrates the general transition from $3 \mathrm{D}$ via $2 \mathrm{D}$ to $1 \mathrm{D}$ and from Scale 7 to 1 at the same time, including the intermediate interpolated structures. In Fig. 7 we show a specific transition

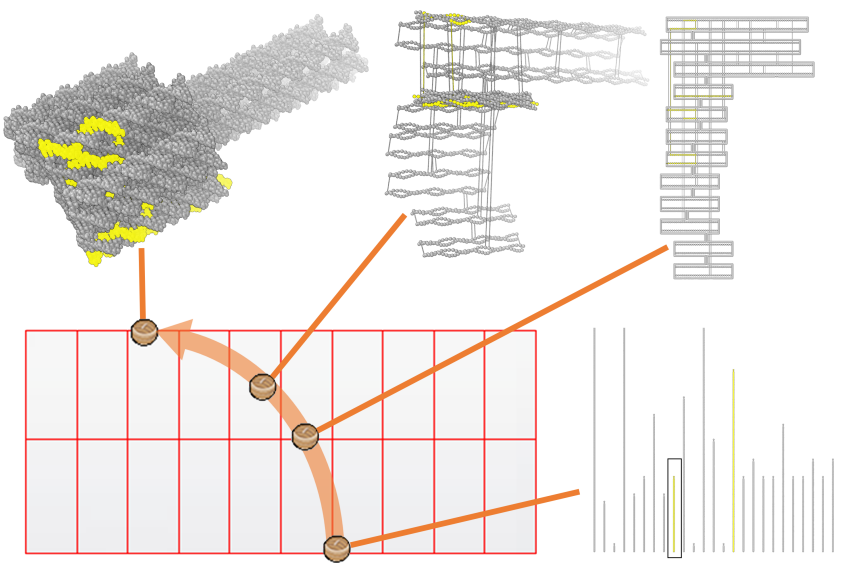

Figure 7: After two strands are selected in 1D, we transition along the orange arrow and show examples of the changing representation.

along the orange arrow, going from $d=1, s=6$ to $d=3, s=2.3$, and depict some of the interpolated representations. This example demonstrates how we transition from an abstract to a concrete representation of the same structure, allowing the scientists to visually connect the involved parts.

Transitioning from 2D to 3D thus enables the viewer to understand how the structure folds together from the schematic display to its 3D shape. Transition from 2D to 1D depicts how the structural design decomposes into the single strands. Combining transitioning across dimension with transitioning across scales enables the scientists to seamlessly move between any two points of the abstraction space, and to take specific paths in this space to intuitively explore the data. The interpolation guarantees a uniform positional change of the elements. Obviously, the current interpolation of positions does not realistically depict the movement and folding of strands, which would require large-scale molecular dynamics simulations. The simplified transition, however, illustrates relationships and allows the viewer to mentally integrate between any representations.

\subsection{Cross-Dimension and Scale Highlighting}

In order to boost the coherent connection between the different dimensions and scales, we enable the user to highlight structures at any location in the abstraction map. The viewer can either manually select the elements or automatically highlight certain features and then observe how they transition into other representations.

Manual Highlighting. We allow the viewer to mark elements of interest (atoms, nucleotides, single strands, double strands) at any position in the abstraction map, which we highlight (e. g., in yellow). We then color context elements with a visually less salient color (e. g., in gray) to increase the focus on the highlighted elements. Fig. 7 shows how two strands are selected at $d=1, s=6$ in the abstraction map and how they are depicted in other locations in the map as the user transitions along the marked path. The transitions allow the user to observe where the particular strands end up in the 2D schematics and the 3D structure. The figure also illustrates the transition across scales. In the detailed atomic view, the atoms that are part of the strands are highlighted as well.

Automated Highlighting. Interesting features that appear re- 

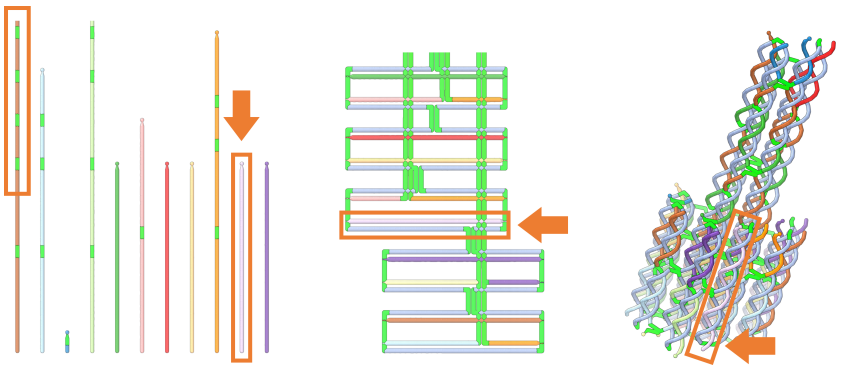

Figure 8: Crossovers are highlighted in green. Short segments between crossovers could have an adverse effect on the stability, which is best observed in $1 D$.

peatedly in the entire structure can also be detected automatically. Crossovers, for instance, are fundamental in DNA nanotechnology (Sect. 2). A relatively high number of crossovers has an adverse effect on the stability of the strand binding, as the segments could have low stickiness. These crossovers can easily be observed in 3D but visual occlusion may make it difficult to gain an overview. This overview is instead provided by the $2 \mathrm{D}$ schematics which indicates how well the double helices are held together. In 2D, however, it is difficult to track an individual strand and to see how many crossovers are performed. In contrast, at $d=1$ in our abstraction map, individual crossovers can be easily observed. We compute crossover locations in the structure and then highlight (e.g., in green) the two nucleotides that form the crossover from one double helix to a neighboring one. An example is shown in Fig. 8. This way we do not deemphasize the context-it is important to keep the contextual information encoded here. The number of crossovers can be simply determined and the length of the segments between crossovers can be visually inspected. Usually, long segments tend to bind better, provided that the sequence is complementary. This sequence complementarity is best seen at $d=2, s=(3 \mid 4)$. In Fig. 8 we marked the brown strand that has relatively short segments of only four nucleotides between crossovers. This configuration could potentially compromise the stability of the entire structure. The arrow points to the pink strand, which has no crossovers and does not contribute to binding adjacent double helices together. The pink strand could thus be a good candidate for connection with another strand. These features are best discovered in our abstraction map at $d=1$.

\subsection{Abstraction-Adaptive Modifications}

Structural modifications are an important part in the workflow of the domain scientists. Deleting, breaking, connecting, and concatenating are basic operations that allow them to carry out advanced modifications of a structure. We provide such structural modifications at any point in the abstraction map with a well-defined behavior and scope of the respective effect. While Miao et al. [MDLS*18] proposed Scale-Adaptive Modifications, we extend these modifications across dimensions, enabling users to modify a structure in 2D and 1D layouts. A particular task can be carried out at the scale where it makes most sense and the system propagates automatically the modification not only across scales but also across dimensions (abstraction-adaptive). Next, we demonstrate two tasks for which this operation across dimensions is particularly useful.
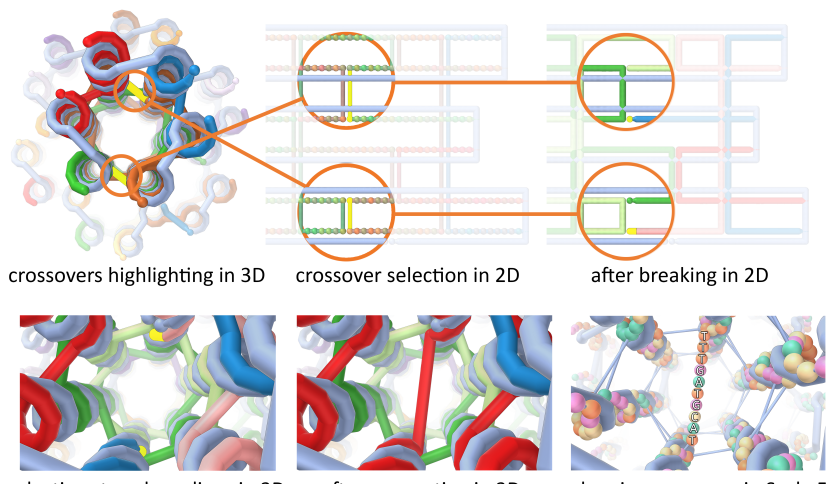

selecting strands endings in 3D

after connecting in 3D

showing sequence in Scale 5

Figure 9: The addition of a bridging strand inside the nanotube requires work in different dimensions and scales. Top row: crossovers are broken in $2 D$. Bottom row: after breaking, the broken strands are connected with a specific sequence.

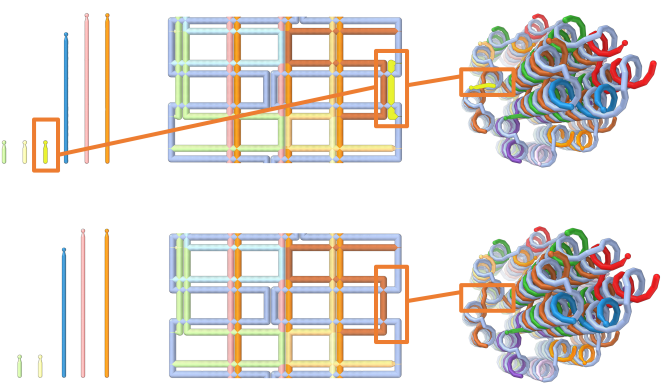

Figure 10: Deleting of undesired short strands. Left: a sort by length in $1 D$ reveals the short strands. Top row: one of them is selected in $1 D$ and deleted. The same strand is shown in $2 D$ and $3 D$. Bottom row: representations in all dimensions show that the strand is removed.

In Fig. 9 we demonstrate a task where a bridging strand is added to the inner part of the nanotube. First we depict the nanotube in 3D $(d=3, s=7)$ and highlight the two crossovers that need to be broken. Due to the visual occlusion in 3D, which we have just described, we select the crossovers in 2D $(d=2, s=5.5)$ and break them. We then create a new connection of the nucleotides at the broken crossover location and we add a sequence of ten nucleotides (TACGTAGTTT) in-between in 5' to 3' direction.

In Fig. 10 we demonstrate the removal of a particularly short strand that does not contribute to the stability of the structure. According to the domain scientists, these short strands can cause problems during the self-assembly as they have a high probability to bind to regions and would be in the way of the intended folding. In addition, they generate costs during the synthesis. To identify short strands, the scientists can now sort the strand by length and quickly see that there are three staple strands of only length three. They can select one strand and depict it in the other dimensions for demonstration purposes. After removal, Fig. 10 shows that the scaffold strand is unpaired at the respective location. However, the brown staple strand can not be extended along the helical axis because crossovers 


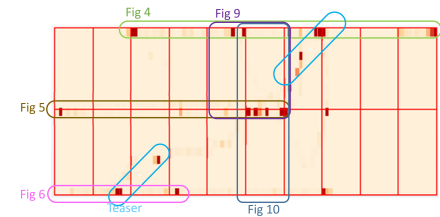

(a) Frequently visited places on the abstraction map. We marked the places that were used to create the figures in this work.

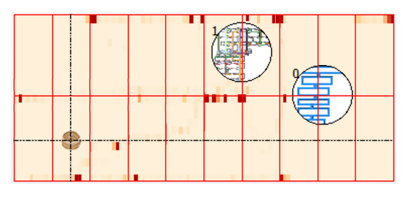

(b) Snapping to the discrete dimension and scale or current location. The thumbnails show the explicit viewpoints defined by the user.
Figure 11: We augment the DimSUM with additional information. The heatmap depicts the accumulated time a user spent at a particular point. The time in milliseconds is mapped to a

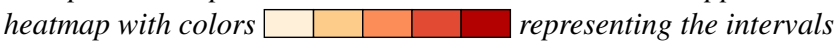
$[0 ; 1000),[1000,2000),[2000,3000),[3000,4000),[4000,+\infty)$.

cannot be formed at locations where the helical twists move away from each other. This can be seen in our 3D representations.

\section{Interaction and Navigation in the Abstraction Map}

As we provide a large set of representations, we developed a twodimensional widget that allows the user to easily change representation by moving an icon on the abstraction map. Our abstraction map not only serves as a concept to organize the transitions across semantic scales and dimension, but we use it directly as a navigation widget to access the abstraction space-in a similar way as done previously by Mohammed et al. [MAAB*18]. In contrast to their approach for connectomics, our abstraction map is completely continuous, despite the transitions between spatial 3D representations and abstract 2D and 1D depictions. In addition, we augment the DimSUM abstraction map with a heatmap to mark interesting, frequently visited locations, which indicate particularly relevant representations for a given dataset or task. In a collaborative setting, such highlighting allows team members to understand each others interest when working on the same dataset. The heatmap also provides individual users with a way to track their own interaction and data exploration patterns. Finally, it allows the scientists to understand which parts of the abstraction space they have not yet investigated in detail, to look for potentially interesting representations.

The heatmap in Fig. 11a, for example, depicts the places that we visited to create the figures in this paper, highlighting the distinct abstractions that we used. It shows that we not only visited the discrete dimensions and scales but also the space in-between. For the teaser in Fig. 1, for instance, the transition is clearly marked, indicating the frequent use of this space.

We also support the navigation in the abstraction map by allowing users to snap the cursor to either a discrete dimension (with the $d$ $k e y)$ or a discrete scale (s-key) if the cursor lies within a radius of 0.25 units. Alternatively, users can snap the curser to its current non-integer location ( $g$-key). The display of dotted vertical and horizontal lines (Fig. 11b) further assists this interaction. Finally, we allow users to explicitly mark locations by placing view points in the abstraction map, enabling them to come back to this location at a later point in time. As shown in Fig. 11b, we add thumbnails to visually mark such locations.

\section{Realization / Implementation}

Our system has to effectively integrate all the data required for the visualization in the various dimensions and scales. We employ a model that hierarchically represents the strands, the base pairs, the nucleotides, and atomic details, which we describe next. We initialize this model with the original caDNAno 2D diagrams, from which we derive all the information, such as $2 \mathrm{D}$ and $3 \mathrm{D}$ positions.

CaDNAno diagrams are based on a lattice, which covers a plane. As design principle it is assumed that the helical axis of all dsDNA are perpendicular to this plane. On the lattice the positions of the virtual helices are defined. Double strands can be formed by conducting the scaffold strand through them. In the caDNAno file format it is specified which virtual helices are in fact used in the design by indicating their rows and columns in the lattice. The positions occupied by scaffold nucleotides and/or staple nucleotides within every virtual helix and the routing of every strand across the virtual helices is also specified. The used lattice and the sequence of the scaffold must be provided by the user as it is not contained in the data. For the datasets in this paper we take the standard m $13 \mathrm{mp} 18$ bacteriophage DNA as scaffold sequence as this is used by our domain scientists. However, any other sequence in the FASTA-format can be input into our system.

The 3D, 2D, and 1D layouts depend on the positions of nucleotides. Each nucleotide defines a position in each dimension. From caDNAno we derive the $2 \mathrm{D}$ conformations. We then determine the corresponding $3 \mathrm{D}$ positions using the $2 \mathrm{D}$ conformation and the lattice information. We can, finally, compute the corresponding 1D locations from a side-by-side placement of single strands. We use the sorting criteria described in Sect. 4 as the different ssDNA are now completely described according to our model. In all dimensions we make use of the geometric properties of a typical double helix to achieve models as realistic as possible. Furthermore, to generate an all-atom 3D model we use idealized base pairs [LO03]. We generate additional scales by aggregating data and, for the transition along the scale-axis, we linearly interpolate colors, shape parameters, and positions from one discrete scale to the next, both as described by Miao et al. [MDLS*18]. For the transition along the dimension-axis, we interpolate positions. By combining both interpolations we generate a morphing-like transformation between any two points in the abstraction map.

We implementated our system using the SAMSON framework [NAN16], a platform for fast prototyping in computational nanoscience. We used its capability of rendering geometric primitives such as spheres and cylinders for depicting the primitives in our visualizations. We implemented the user interface using the $Q t$ framework [The17]. For calculating the melting temperature and Gibbs free energy, we used the ntthal package from the Primer 3 software [WRU* $16, \mathrm{UCK}^{*} 12$ ]. We tested our system on a laptop with an Intel Core i7 CPU and an Nvidia GTX 1060 GPU. For the datasets shown in this work, we achieve 20 fps or more.

\section{Results and Discussion}

Because we realized our work in close collaboration with domain experts (Sect. 4), we could directly derive their requirements by observing their workflow. The case studies, which we describe next, 


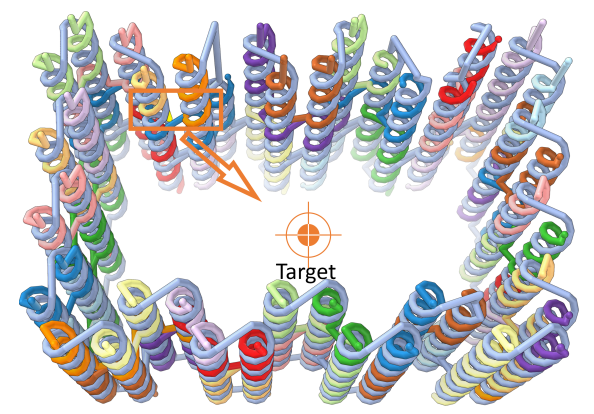

(a) Nanocage. A staple needs to be elongated towards the target in the center.
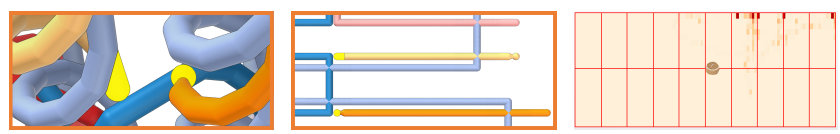

(b) Two candidates for (c) The same strands are (d) The logged abstracelongation. On the left, depicted in 2D, which tion map depicts which the helical twist turns to- does not provide means locations expert C4 viswards the targeted cen- to estimate the helical ited in order to anater. On the right, the twists and positions of lyze the structure and to strand turns away. strands. carry out this task.

Figure 12: Demonstration of the detection of specific surface strand.

are the result of focus group discussions as well as of feedback we received during the regular progress reports to the team. We further developed the case studies in two final sessions in which we provided the domain scientists with our system, observed their interaction, and logged their behavior in the DimSUM abstraction map. The results demonstrate the effectiveness and efficiency of our approach. Furthermore, the experts could gain insights about their work that were not possible before, in particular with respect to the missing connection between $3 \mathrm{D}$ and $2 \mathrm{D}$ representation.

\subsection{Surface Strand Analysis}

A main challenge in the design of nanostructures is that scientists need to rely on realistic estimations of distances and location of the structural elements. Fig. 12 depicts a cage that we use to demonstrate the case. Here, a common task is to identify the strand endings that are on the inside of the cage. These endings are candidates for elongation. Such identification tasks are challenging when designing and modifying DNA nanostructures. With the existing tools such as caDNAno it is difficult to determine the strand endings according to expert C2: Not only their locations but also their directions of the helical turn have to be carefully considered.

Therefore, expert $\mathrm{C} 2$ suggested to carry out this task in our system. We asked expert $\mathrm{C} 4$, who is very experienced with the traditional tool, to find the surface strands and provide us with feedback. She investigated the DNA origami cage with dimensions of $200 \AA \times 200 \AA$ a dataset with which she was not familiar before. It contains 56 staple strands and one scaffold strand with 2197 nucleotides, aligned in a honeycomb lattice. Her goal was to identify those strand endings that could potentially be elongated to create a connection towards a target in the center of the cage, as illustrated in Fig. 12a.

While she was familiar with our project, she did not actively use our system before. We first gave her an introduction into the user interactions and explained the visualization concepts. Then she used our system to explore the data and become familiar with it. We logged her interactions during task executing on the DimSUM abstraction map, and we show the resulting heatmap in Fig. 12d. As it is apparent in the figure, the $3 \mathrm{D}$ representations at the higher scales were the most interesting ones for identifying the surface strands. According to the expert, she was able to have a straightforward view of the spatial model in 3D and to relate it back to the familiar 2D view. She stated that one big advantage of our approach is that she could get an overview of all possible strand endings and quickly identify those on the inside of the cage. She quickly found two candidates, which we highlight in Fig. 12a. We give a zoomed-in image in Fig. 12b, which shows that these endings are located on the bottom of the lower double helices. She realized that, although both strands end at the bottom, only the left yellow strand turns towards the target in the center of the cage, whereas the right orange one turns away and is, hence, not an ideal candidate for elongation. According to $\mathrm{C} 4$, this precise analysis in the planning stage enables her to conduct a time-efficient and accurate in silico design and thus reduces the probability of introducing errors that compromise the successful assembly of the nanostructure in the wet lab later. Finally, she transitioned to the 2D representation as shown in Fig. 12c, in order to compare the 2D depictions, which she is familiar with.

This case demonstrates how our approach can assist the domain scientists in tasks that require a good understanding of both the schematic design and the resulting spatial layout. This makes the workflow in such cases much simpler. We also learned that navigating through the $3 \mathrm{D}$ scene can pose a challenge to some users such as $\mathrm{C} 4$, as they might need prolonged time to familiarize with $3 \mathrm{D}$ navigation. Later we discussed this case with $\mathrm{C} 2$ and he suggested to automatically detect the surface endings in the future and then to highlight them in our visualization. According to $\mathrm{C} 2$, however, our system allowed the scientists to perform the manual identification very well in comparison with existing tools, where the detection of these surface strands is a tedious task.

\subsection{Parametrized Generation of Structural Motifs}

One domain scientist (C3) has been using our system intensively throughout the development and has embedded it into her workflow, starting at an early point in time. She specializes on the computational design of DNA nanostructures and her goal is to develop methods for the parametrized generation of structural motifs and the computational design of functional nanostructures. The reliance on visualization and the exploratory nature of her work, however, requires her to analyze her results with different methods. She provided regular input and was therefore actively involved in the conceptual development of this work.

In a final interview we asked her again to explain how the system supports her and logged her interaction with our system on her own machine. She loaded the robot/man dataset, used in the work of Castro et al. [CKK $\left.{ }^{*} 11\right]$, to demonstrate how our new approach helped in her tasks. As shown in Fig. 13a, she first selected the shoulder of the man and then transitioned from $3 \mathrm{D}$ to $2 \mathrm{D}$ to observe where this part of the structure is located in the 2D diagram. She stated that the $3 \mathrm{D}$ spatial model is necessary for her to understand the 2D schematics, 


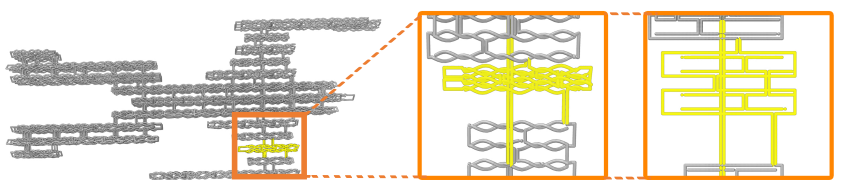

(a) Transition from $3 D$ to $2 D$.

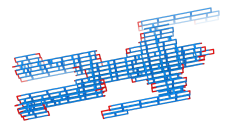

(b) $s=8$

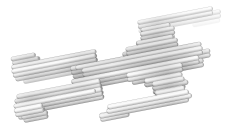

(c) $s=9$

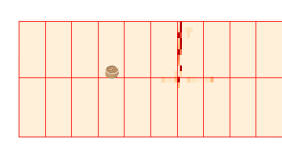

(d) Abstraction map.
Figure 13: Using transition to mentally link between $3 D$ to $2 D$.

which is sometimes challenging due to the loss of spatial context. According to her, this problem is solved because she can now relate between these two representations in DimSUM. In the heatmap of the logged session (Fig. 13d) we can observe the corresponding frequent transitions. As she is already experienced with our system, she could quickly navigate to the appropriate representation using the proposed interaction techniques. Her feedback regarding transitions between dimensions focused on the development of new algorithms for DNA origami design. The visualization of these transitions made easier for her to understand the DNA origami method in general. She also noted that it can greatly reduce the amount of time spent on debugging DNA nanostructure designs.

Overall, she described that the ability of visualizing the same structure in many dimensional and semantic representations and having the ability to relate between these representations helped her to visually examine the results to validate the correctness of the computational methods she developed. She stated that, for example, a faulty in silico design will produce incorrect foldings in the laboratory. Using our system and being able to visually examine the in silico design in the proposed manner, she could find errors in the computational methods. She stated that this saves costs and also time spent for assembling potentially erroneous structures.

\section{Conclusion and Future Work}

In this paper, we discuss the application of visual abstraction to DNA nanotechnology. DimSUM, our two-axes abstraction space goes beyond the work of Mohammed et al. [MAAB*18], which actually encodes geometry and dimensionality on a single axis. We advance the concept of abstraction by demonstrating that dimensionality is an important axis, where different layouts provide specific advantages in analyzing and modifying complex data.

Because we integrated the visual abstractions into an interactive widget we facilitate seamless transitions. This possibility to mentally integrate all representations in our abstraction space, allows domain scientists to include our visualization concept into their workflow, and to improve it. Another contribution includes the use of the DimSUM abstraction map as interaction widget. Traces of user interaction can be taken to optimize visualization settings for particularly focused tasks that do not use the entire visual abstraction space. We could also apply user traces in a systematic design of custom MCV systems. Only a task-dependent subset of views that have been visited during the interaction will then form the simplified visual interface. A MCV could be advantageous if it allows the user to immediately understand the implications of a modification in views other than the one he or she is currently operating with.

Our DimSUM approach can potentially be generalized to data based on linear structures. The key idea is to share the complexity of data among several layouts and scales, each representation highlighting a certain aspect of the data. As noted in Sect. 3, abstractions of linear structures are important, e. g., in cartography. Here, line representation of streets are continuously generalized depending on the distance to the viewer. Blood vessels also share traits with DNA w.r.t. their different semantic representations (volume, surface, centerlines, etc.) and are typically abstracted to 2D depictions. Another example are fibrous structures in material sciences, where defective fibers could be singled out easily in a 1D arrangement. For the future, it could be of great interest to explore the possibility of localized glimpses into other dimensions and scales while keeping the context—similar to the magic lens [BSP*93] metaphor.

Furthermore, we showed how our visualization concept of interactive abstraction is helpful to the domain of DNA nanotechnology. We provide domain scientists with a proof-of-concept implementation that is already in the daily workflow of some experts, (e.g., C3). One of the limitations of our approach is that we currently are only implementing model modifications. We do not yet provide from-scratch modeling of new designs. Another drawback is that we are not using the positional data available from simulations. DNA origami structures could exhibit twists and bends in their shape model, for example. We want to incorporate these structural predictions in the future to provide better approximations. A more general limitation is that large datasets are increasingly difficult to inspect: elements can become too small for an effective work if viewed from far away. This scalability issue is not necessarily a drawback specific to our approach, but of DNA diagrams in general. Furthermore, users have remarked that the transitions from 2D to $1 \mathrm{D}$ are not easy to follow when working with larger datasets. Nonetheless, this concern could be addressed in future work that looks at the design approaches in DNA origami in general to provide a scalable and efficient workflow. Our next research opportunity is to extend the presented work to general DNA nanostructures, including, but not limited to, wireframe structures [VRZ* $\left.16, \mathrm{BMG}^{*} 15\right]$, which exhibit a great potential for applications under physiological conditions. In the near future, the implementation of our approach will be released as part of a open-source software toolkit.

\section{Acknowledgments}

This project has received funding from the European Union's Horizon 2020 research and innovation programme under grant agreement No 686647. This work was also partially funded under the ILLUSTRARE grant by FWF (I 2953-N31), ANR (ANR-16-CE910011-01), and the WWTF (VRG11-010). This paper was partly written in collaboration with the VRVis Competence Center. VRVis is funded by BMVIT, BMWFW, Styria, SFG and Vienna Business Agency in the scope of COMET - Competence Centers for Excellent Technologies (854174) which is managed by FFG. We thank Yasaman Ahmadi and Tadija Kekic for providing valuable input to this work and also David Kouril who created the submission video. 


\section{References}

[AS01] Agrawala M., STOLTE C.: Rendering effective route maps: Improving usability through generalization. In Proc. SIGGRAPH (2001), ACM, New York, pp. 241-249. doi: 10.1145/383259.383286 3

[BC87] BeCKer R. A., Cleveland W. S.: Brushing scatterplots. Technometrics 29, 2 (May 1987), 127-142. doi: 10.1080/00401706.1987.10488204 4

[BMG*15] Benson E., Mohammed A., Gardell J., Masich S., CzEIzler E., ORPONEN P., HöGBERG B.: DNA rendering of polyhedral meshes at the nanoscale. Nature 523, 7561 (2015), 441-444. doi: 10. 1038/nature14586 3, 10

[BMMS91] Buja A., McDonald J. A., Michalak J., Stuetzle W.: Interactive data visualization using focusing and linking. In Proc. Visualization (1991), IEEE Computer Society, Los Alamitos, pp. 156-163. doi: 10.1109NISUAL.1991.175794 4

[BSP*93] Bier E. A., Stone M. C., Pier K., Buxton W., DeRose T. D.: Toolglass and magic lenses: The see-through interface. In Proc. SIGGRAPH (1993), ACM, New York, pp. 73-80. doi: 10.1145/166117. 16612610

[CD16] Conway N., Douglas S.: caDNAno. Website: http:// cadnano.org/, 2016. Visited in March 2017. 2

[CdBvK05] Cabello S., De Berg M., van Kreveld M.: Schematization of networks. Computational Geometry 30, 3 (Mar. 2005), 223-238. doi: 10.1016/j.comgeo.2004.11.002 3

[CG07] CiPRIANo G., Gleicher M.: Molecular surface abstraction. IEEE Transactions on Visualization and Computer Graphics 13, 6 (Nov./Dec. 2007), 1608-1615. doi: 10.1109/TVCG.2007.70578 3

[CKK*11] Castro C. E., Kilchherr F., Kim D. N., Shiao E. L., Wauer T., Wortmann P., Bathe M., Dietz H.: A primer to scaffolded DNA origami. Nature Methods 8, 3 (Feb. 2011), 221-229. doi: 10. 1038/nmeth.1570 9

[DMT*09] Douglas S. M., Marblestone A. H., TeerapitTAYAnon S., VAzQuez A., Church G. M., Shih W. M.: Rapid prototyping of 3D DNA-origami shapes with caDNAno. Nucleic Acids Research 37, 15 (Aug. 2009), 5001-5006. doi: 10.1093/nar/gkp436 2, 3

[Ise13] IsENBERG T.: Visual abstraction and stylisation of maps. The Cartographic Journal 50, 1 (Feb. 2013), 8-18. doi: 10.1179/1743277412Y. 00000000073

[JDL09] JIANU R., DEMIRALP C., LAIDLAW D.: Exploring 3D DTI fiber tracts with linked 2D representations. IEEE Transactions on Visualization and Computer Graphics 15, 6 (Nov. 2009), 1449-1456. doi: 10.1109/TVCG. 2009.1414

[LMAPV15] Le Muzic M., Autin L., Parulek J., Viola I.: celIVIEW: A tool for illustrative and multi-scale rendering of large biomolecular datasets. In Eurographics Workshop on Visual Computing for Biology and Medicine (2015), Eurographics Association, Goslar, Germany, pp. 6170. doi: $10.2312 / \mathrm{vcbm} .201512093$

[LO03] LU X., OLSON W. K.: 3DNA: A software package for the analysis, rebuilding and visualization of three-dimensional nucleic acid structures. Nucleic Acids Research 31, 17 (Sept. 2003), 5108-5121. doi: 10. 1093/nar/gkg680 8

[MAAB*18] Mohammed H., Al-Awami A. K., Beyer J., Cali C., Magistretti P., Pfister H., Hadwiger M.: Abstractocyte: A visual tool for exploring nanoscale astroglial cells. IEEE Transactions on Visualization and Computer Graphics 24, 1 (Jan. 2018), 853-861. doi: 10. 1109/TVCG.2017.2744278 4, 8, 10

[MDLS*18] Miao H., De Llano E., Sorger J., Ahmadi Y., KeKic T., ISENBERG T., GRÖLlER M. E., BARIŠIĆ I., VIOLA I.: Multiscale visualization and scale-adaptive modification of DNA nanostructures. IEEE Transactions on Visualization and Computer Graphics 24, 1 (Jan. 2018), 1014-1024. doi: 10.1109/TVCG.2017.2743981 2, 3, 5, 6, 7, 8

[NAN16] NANO-D, INRIA: SAMSON - Software for adaptive modeling and simulation of nanosystems. Website: https:// samson-connect. net, 2016. Visited December 2017. 8
[PJR*14] PARULEK J., JÖNSSON D., ROPINSKI T., BRUCKNER S., YNNERMAN A., VIOLA I.: Continuous levels-of-detail and visual abstraction for seamless molecular visualization. Computer Graphics Forum 33, 6 (Sept. 2014), 276-287. doi: 10.1111/cgf.12349 3

[Rob07] RoBERTS J. C.: State of the art: Coordinated multiple views in exploratory visualization. In Proc. CMV (2007), IEEE Computer Society, Los Alamitos, pp. 61-71. doi: 10.1109/CMV.2007.20 4

[Rob12] RoBERTS M. J.: Underground Maps Unravelled: Explorations in Information Design. Maxwell J. Roberts, 2012. 3

[Rot06] RothemUND P. W.: Folding DNA to create nanoscale shapes and patterns. Nature 440, 7082 (Mar. 2006), 297-302. doi: 10.1038/nature04586 $1,2,3$

[See82] SEemAn N. C.: Nucleic acid junctions and lattices. Journal of Theoretical Biology 99, 2 (Nov. 1982), 237-247. doi: 10.1016/0022-5193(82) 90002-9 1

[SH04] SantaluCia J., Hicks D.: The thermodynamics of DNA structural motifs. Annual Review of Biophysics and Biomolecular Structure 33, 1 (2004), 415-440. doi: 10.1146/annurev.biophys.32.110601.141800 6

[SMR*17] Sorger J., Mindek P., RAUTEK P., Gröller M. E., JOHNSON G., VIOLA I.: Metamorphers: Storytelling templates for illustrative animated transitions in molecular visualization. In Proc. SCCG (2017), Brno University of Technology, Czech Republic, pp. 27-36. 4

[STKD12] Semmo A., Trapp M., Kyprianidis J. E., Döllner J.: Interactive visualization of generalized virtual 3D city models using levelof-abstraction transitions. Computer Graphics Forum 31, 3pt1 (June 2012), 885-894. doi: 10.1111/j.1467-8659.2012.03081.x 3

[The17] The QT Company: Qt. Website: https://www.qt.io/, 2017. Visited December 2017. 8

[TMB02] Tversky B., Morrison J. B., Betrancourt M.: Animation: Can it facilitate? International Journal of Human-Computer Studies 57, 4 (2002), 247-262. doi: 10.1006/ijhc.2002.1017 4

[UCK*12] Untergasser A., Cutcutache I., Koressaar T., Ye J., FAircloth B. C., Remm M., Rozen S. G.: Primer3-New capabilities and interfaces. Nucleic Acids Research 40, 15 (2012), e115:1-e115:12. doi: $10.1093 / \mathrm{nar} / \mathrm{gks} 5968$

[vdZLBI11] VAN DER ZWAN M., LUEKS W., BEKKER H., ISENBERG T.: Illustrative molecular visualization with continuous abstraction. Computer Graphics Forum 30, 3 (May 2011), 683-690. doi: 10.1111/j.1467-8659.2011. 01917.x 4

[VI18] Viola I., Isenberg T.: Pondering the concept of abstraction in (illustrative) visualization. IEEE Transactions on Visualization and Computer Graphics 24 (2018). To appear. doi: 10.1109/TVCG.2017.2747545 3 ,

[VRZ*16] VEnEZIANO R., RATANALERT S., ZHANG K., ZHANG F., YAN H., ChIU W., BATHE M.: Designer nanoscale DNA assemblies programmed from the top down. Science 352, 6293 (June 2016), 1534:11534:15. doi: 10.1126/science.aaf4388 10

[WC53] Watson J., CRICK F. H. C.: Molecular structure of nucleic acids; a structure for deoxyribose nucleic acid. Nature 171 (Apr. 1953), 737-738. doi: 10.1038/171737a0 2

[Wil08] WiLLs G.: Linked data views. In Handbook of Data Visualization, Chen C.-h., Härdle W., Unwin A., (Eds.). Springer, Berlin/Heidelberg, 2008, ch. II.9, pp. 217-241. doi: 10.1007/978-3-540-33037-0 104

[WRU*16] WHITEHEAD INSTITUTE FOR BIOMEDICAL RESEARCH, Rozen S., Untergasser A., Remm M., KoressaAr T., SkaletSKY H.: Primer3. Website: http://primer3.sourceforge. net/, 2016. Visited in March 2017. 8

[WTLY12] Wu H.-Y., TAKahashi S., Lin C.-C., Yen H.-C.: Travelroute-centered metro map layout and annotation. Computer Graphics Forum 31, 3 pt1 (2012), 925-934. doi: 10.1111/j.1467-8659.2012.03085.x 3 